\title{
Acanthamoeba Keratitis - The Quest for a Better Prognosis
}

\author{
LINDA FICKER \\ London
}

\section{Epidemiology}

Acanthamoeba keratitis is recognised to be a growing clinical problem and the increased incidence is associated with contact lens wear, both hard and soft. ${ }^{1}$ Acanthamoeba polyphaga was first reported as a corneal pathogen in $1973,{ }^{2}$ but prior to that the diagnosis may have been missed for want of specific investigations. Acanthamoeba castellani, A. Colbertsoni, A. rhysodes and $\mathrm{A}$. hatchetii have subsequently been identified as corneal pathogens. ${ }^{2-6}$

Ashton et $\mathrm{al}^{7}$ reviewed 756 corneal specimens of keratitis and ulcerative keratitis sent to the Institute of Ophthalmology between 1960 and 1975, specifically to determine the incidence of Acanthamoeba keratitis during that period. Haematoxylin and eosin preparations were restained with silver methenamine (106 cases) or immunofluorescent antibody (20 cases) if cells resembling cysts or trophozoites were seen. Acanthamoeba were not found, but it has since been recognised that immunofluorescent, immunoperoxidase and lectin stains are considerably more sensitive in detecting possible cysts and trophozoites. ${ }^{8-10}$ More recently, Cohen et al found acanthamoebae in two of eight corneal specimens from patients grafted for idiopathic keratitis between 1974 and 1983. ${ }^{11}$

The first case report involved a rancher who suffered minor corneal trauma while working with bales of straw ${ }^{2}$ and similar histories were obtained for most of the early cases described periodically in the literature. ${ }^{12-18}$ In 1985 the number of reported cases increased tenfold and over $80 \%$ were contact lens wearers. Of 51 contact lens-related cases, reported between 1974 and 1986, 75\% occurred with daily wear and $14 \%$ with extended wear soft contact lenses. ${ }^{1}$ Contamination was attributed to the use of home-made saline by $62 \%$ and tap water by
$16 \%$ of the patients. Contamination of contact lens care systems by acanthamoeba has been increasingly documented and has included bacteria (generally Gram negative rods) and fungi in combination with acanthamoebae. ${ }^{19}$ Most published cases lived in warmer climates or became symptomatic during the summer.

Many contact lens disinfection techniques have been found ineffective in destroying acanthamoebae (3\% hydrogen peroxide, thimerosal-edetate chlorhexidine-edetate, edetate disodium thiomersal and potassium sorbate) ${ }^{20}$ Heat disinfection, however, is effective for $\mathrm{A}$. castellani and A. polyphaga. Where heat disinfection is not possible, it is recommended that clinical disinfection should be performed cleanly and with commercially prepared preserved solutions. These are available in small volumes which may be discarded weekly to minimise the risk of contamination. ${ }^{1}$

Acanthamoebae are ubiquitious, free-living in air, soil and water (including chlorinated swimming pools) with peak prevalence in warmer weather. ${ }^{21}$ They undergo transformation from an active trophozoite to a dormant cyst which is resilient to a wide range of environmental extremes. Cysts remain viable after exposure to temperatures between $-20^{\circ} \mathrm{C}$ and $42^{\circ} \mathrm{C}^{22-24}$ and through a $\mathrm{pH}$ range from 3.9 to $9.75 . .^{25}$ Thus acanthamoebae have the credentials of opportunist pathogens. In several cases of acanthamoeba keratitis the pathogen was also isolated from the suspected environmental source including well water, tap water and hot tub water. ${ }^{2,17,26}$ The precise pathogenesis for keratitis has not, however, been elucidated.

Humoral immunity has been reported for pathogenic acanthamoebae suggesting subclinical exposure of the study populations. ${ }^{27}$ 
Although they may colonise the nasopharynx, ${ }^{28-30}$ there is no evidence to suggest this plays an aetiological role in keratitis. It is interesting that granulomatous amoebic meningoencephalitis usually occurs in debilitated and immunocompromised patients. ${ }^{31}$ while disseminated acanthamoeba infection occurs in AIDS patients without a granulomatous response. ${ }^{32}$ The cornea is relatively isolated from immune surveillance and the nature of the immune response to acanthamoebae in the cornea may be an important factor in determining its susceptibility to treatment. Acanthamoeba keratitis was diagnosed in nine patients (11 eyes) at Moorfields Eye Hospital between July 1986 and November 1987. These patients did not show a seasonal distribution for the onset of symptoms, but two bilateral cases had their onset in the warmer climates of Ecuador and Crete. Although one patient gave a history of trauma from the branch of a bush, the remainder were contact lens wearers; five daily wear soft lenses (four patients), three Saturn lenses (two patients) and two hard lenses. One soft lens wearer (with bilateral disease) wore his lenses swimming in a chlorinated pool, two made solutions from salt tablets and distilled water and one of these resorted to lenses stored for some time in homemade solution while having the current lenses professionally cleaned. The two Saturn lens wearers had used water from streams and water troughs while travelling. One hard lens wearer used no solutions, while the other resumed contact lenses after several months without prior disinfection. These patients represented a dramatic increase in the number of cases of acanthamoeba keratitis, compared with previously sporadic cases, seen at Moorfields, reflecting both the risk from contact lenses and a growing awareness of the variety of clinical manifestations of the disease.

\section{Clinical presentations}

In many cases there is delay in making the diagnosis of acanthamoeba keratitis since it may masquerade as herpetic keratitis or may be presumed bacterial. ${ }^{33}$ Fungal keratitis has been suspected when corneal satellite lesions occur. ${ }^{4,34}$ Where contact lens-related keratitis does not appear typical of either bacterial or viral infection, and particularly if there is an indolent course, acanthamoebic infection should be considered in the differential diagnosis. Among early reports initial clinical improvement with antiviral or antibiotic treatment was a factor in diagnostic delay.

Relatively few cases have been recognised early in the course of the disease. The early signs described have included superficial punctate keratitis and microcystic oedema ${ }^{1,11,26}$ with intact epithelium initially, but occasionally progressing to dendritic epithelial ulceration. $1,11,9,35$ Later, anterior stromal infiltrates become increasingly prominent, curvilinear and gradually forming a paracentral ring surrounded by clear cornea. ${ }^{12}$ The overlying epithelial defect may heal, but frequently undergoes recurrent breakdown. Ring infiltration often heralds stromal thinning and when progressive this has been interpreted as incipient perforation. Perforation has frequently been described as a complication of progressive disease $e^{4,6,11,12}$ and Descemetocoele formation is now widely considered an indication for penetrating keratoplasty. In some cases the infiltrate is never observed in the ring configuration. ${ }^{6,11,18,36}$ There can be considerable peripheral encroachment of new vessels on the cornea.

Occasionally perineural infiltrates have been observed ${ }^{37}$ progressing centrifugally towards the corneal periphery. There appears to be a predilection of acanthamoeba for nerve tissue. ${ }^{38}$ Corneal sensation has been reduced in some reported cases, but is infrequently documented. Nerve involvement is probably the basis for the severe pain typical of this disease. Narcotic analgesia has been required in many instances and there has been concern about narcotic addiction. Rarely, nodular scleritis develops in association with corneal infiltrates and is another mechanism for intense pain. ${ }^{4}$

The associated nongranulomatous anterior uveitis generally runs a fluctuating course, probably modulated by topical steroids, with recurrent hypopyon. 2,6,12,15,17,36 The uveitis may be sufficiently severe or chronic to cause cataract and insidious rises in intraocular pressure may further complicate the clinical management. $1,9,11,14,26$

The 11 cases managed at Moorfields Eye Hospital (1986/1987) can be segregated into two groups; one of which includes primary untreated referrals (six eyes) and the other group of treated 
referrals seen later in the course of the disease (five eyes).

Five patients (six eyes) presented to the emergency department within one day to three weeks of symptoms and represent early manifestations of acanthamoeba keratitis. They all complained of considerable pain and photophobia with lid swelling, conjunctival chemosis, redness and reduced vision. On examination the central cornea was preferentially involved with epithelial irregularity and microcystic oedema. Only the two hard lens wearers had focal epithelial defects. Fine subepithelial infiltrates developed with associated stromal oedema around three weeks after the onset of symptoms. This was closely followed by radial keratoneuritis in two cases. At this stage only a few cells were seen in the anterior chamber. Prior to diagnosis the bilateral case in this group developed extensive entothelial plaques of inflammatory debris, with relatively few anterior chamber cells, and corneal oedema.

The other four patients (five eyes) were tertiary referrals. They had been symptomatic for one to three months and their physical signs were probably modified by treatment which included topical steroids in all cases. Four eyes were seen within six weeks and these had considerable corneal oedema with diffuse stromal infiltration and keratic precipitates but relatively few anterior chamber cells, suggesting endothelial inflammation with secondary dysfunction. In one case there were extensive endothelial plaques of inflammatory debris. Superficially there were aggressive new vessels in the corneal periphery and in two cases there was central epithelial loss.

Ring infiltrates were a relatively late feature in seven eyes, all of which had been treated with regimes including topical steroid prior to diagnosis of acanthamoeba keratitis. Ring infiltration appeared between six weeks and five months after the onset of keratitis. Thus the ring infiltrate which was considered to have early diagnostic value ${ }^{17}$ would seem to occur rather late in the clinical course. There was no consistent relationship with anti-amoebic therapy since three were not yet being treated, three had been treated for a few days and one was poorly compliant, resulting in intermittent treatment. Transformation from trophozoite to cyst can be modulated by steroids ${ }^{39}$ and possibly antibiotics, hence these may directly or indirectly influence the inflammatory response. Previous histopathological studies have shown these infiltrates to be characterised by cysts with a rather mild polymorphonuclear response. ${ }^{40}$ This has been our experience and it is interesting that although macrophages may be present, there is a conspicuous absence of lymphoid cells. Corneal sensation was not consistently recorded, but was found to be almost absent in three cases including one with previous keratoneuritis. The other two cases were characterised by diffuse cellular infiltration of the cornea with stromal oedema and endothelial plaques.

\section{Laboratory diagnosis}

Specimens obtained from corneal scrapings of a suppurative keratitis are prepared both for routine microscopy and culture. To investigate the presence of acanthamoeba it is necessary to perform additional specific tests.

While keratitis is active, it is to be expected that trophozoites may feature in the specimens. They are, however, very fragile and rupture on air-drying. The optimal method for preservation of trophozoites has not been established although spray fixatives have been tried. ${ }^{36}$ It is possible to examine trophozoites suspended in Page's saline $^{36}$ by light or phase-contrast microscopy. The trophozoites (16-47 micrometers in length) are slowly moving with acanthopodia and a contractile vacuole which is prominent in the cytoplasm. This movement gives rise to "amoebic" trails on plated media which have been considered diagnostic, but similar trails result from other cell types including corneal epithelial cells and macrophages. ${ }^{41}$

Since the availability of specific immunological tests, diagnostic efforts have not been specifically directed towards identifying trophozoites, because cysts (10-25 micrometers in diameter) are readily identified. Trophozoites have, however, been predominant in some corneal specimens. ${ }^{4}$ and this may reflect their predominance during certain phases of the keratitis. If their survival is compromised by inadequate technique, this may be the basis for some of the negative results in cases which are subsequently shown to be positive for acanthamoeba either by examination of further scrapes or from host cornea excised at kerato- 
plasty. Further efforts to improve preservation of trophozoites in specimens should therefore be made.

Specific immunological labelling of acanthamoeba cysts and trophozoites has allowed rapid and accurate diagnosis from corneal scrapes. ${ }^{9}$ Polyclonal antibodies to both cysts and trophozoites are obtained from rabbit antiacanthamoeba serum. A 1:50 or 1:100 dilution may be used to treat the specimen, after fixation, for 30 minutes. It is then washed with PBS (phosphate-buffered saline), treated with fluorescein-conjugated sheep anti-rabbit IgG for 30 minutes and washed again in PBS. Evans blue is used as a counter-stain. Examination with a fluorescent microscope in blue light reveals fluorescence of any acanthamoebae. ${ }^{35}$

Alternative labelling exists including calcofluor white which is a chemofluorescent dye with an affinity for the cyst wall polysaccharides after fixation. ${ }^{6}$ Two lectins, ConA and WGA, have been found to bind carbohydrate moieties present in the cyst wall and have been fluorescein-conjugated to demonstrate acanthamoebae. ${ }^{10}$

These fluorescein-labelling techniques appear more sensitive than the various stains which may reveal acanthamoebae: Gram, Giemsa, periodic acid-Schiff, Gomori's methenamine silver and Heidenhain's iron haematoxylin being the most useful.

Acanthamoebae are best cultured on nonnutrient agar with an overlay of either Escherichia coli or Klebsiella aerogenes. ${ }^{42}$ The specimen is inoculated onto the surface of this preparation and incubation at $37^{\circ} \mathrm{C}$ should be in a moist environment for two-three days. Examination of the plates is conveniently performed using an inverted microscope to identify motile trophozoites or dormant cysts. Analysis of the success rate of acanthamoeba culturing on standard media, when the diagnosis was not suspected, revealed this to be $12 \%$ compared with $67 \%$ success using special media when the diagnosis was suspected. ${ }^{33}$

Despite the use of these diagnostic techniques, negative scrapes will be obtained if there are few superficial organisms. Acanthamoebae are motile and have the potential for invading the deeper corneal stroma by this mechanism. When host cornea is examined after keratoplasty, it is common to find cysts diffusely distributed through the stroma in association with polymorphonucleur leucocytes and macrophages and, deeper in the stroma, with multinucleated giant cells. $3,12,13,18,43$ Among the cases grafted at Moorfields Eye Hospital (1987-1988) some have been found to have amoebae predominantly in the deep stroma and none superficially. Thus, where corneal scrapes are negative and there is a high index of suspicion, it may be necessary to obtain a corneal biopsy to determine whether acanthamoebae are present. Of the cases managed at Moorfields (1986/1987), ten specimens were treated with immunofluorescent antibody (IFAb) and all 11 were cultured. Six were positively labelled with IFAb and were subsequently successfully cultured. A single specimen was cultured but not treated with IFAb. Hence the results of IFAb and culture were consistent.

The four culture negative cases included one which was being treated after a previous positive culture. This case and another lacked stromal infiltrates at the time corneal scrapes were done. There were, however, plentiful neutrophils seen on microscopy. The other two corneae (one patient) underwent superficial biopsy at the time of axial stromal opacity, again in the absence of active infiltrates.

Contact lens solutions were not available in the majority of cases, but were found to be contaminated with acanthamoeba in the three which were cultured.

Poor compliance resulted, in a single case, in an indolent clinical course during which repeated cultures from corneal scrapes and an aqueous tap were negative at the time of recurrent hypopyons, Eventually total epithelial debridement, four months after the onset of keratitis, gave positive cultures. This case continued to harbour acanthamoebae yet was not consistently culture positive, again suggesting that positive cultures from superficial corneal scrapes are more likely early in the clinical course before invasion of deeper stroma occurs.

A clinical diagnosis was made in three cases (two patients) which required corneal grafts for axial scars, when the eyes were quiet. A definitive histopathological diagnosis was then made using specific IFAb. The finding that acanthamoebae were present only deep in the stroma is the likely reason for the previously negative scrapes. 


\section{Treatment}

In vitro drug sensitivity tests have generated a rational basis for therapy. ${ }^{25,26}$ There are, nonetheless, several caveats including differences between species and strains ${ }^{36,44}$ in addition to $\mathrm{pH}$ dependence ${ }^{25}$ which is likely to be important in inflamed, metabolically active stroma. Furthermore, the pharmacokinetics of these drugs within stroma containing acanthamoebae are not known. It is likely that bound drug will influence efficacy of treatment where topical delivery might be expected to provide amoebicidal concentrations. Although many drugs are effective against trophozoites, there is controversy with respect to cysticidal efficacy. ${ }^{33}$

The diamidines have the lowest minimum inhibitory and amoebicidal concentrations for pathogenic acanthamoebae (1-10 micrograms $/ \mathrm{ml}) .{ }^{36}$ Propamidine and dibromopropamidine isethionate are available as Brolene drops $(0.1 \%)$ and ointment $(0.15 \%)$. Neomycin and paromomycin are the aminoglycosides of choice, with minimum inhibitory concentrations (MIC) less than $10 \mathrm{micrograms} / \mathrm{ml}$. A variety of chemical groups also have amoebicidal activity, but at greater drug concentrations; Polymyxin B and Gramicidin (polypeptides), Venturicidin and Azalomycin (macrolides), Ketoconazole and Clotrimazole (synthetic imidazoles), 5-fluorocytosine and 8-hydroxyqiunolone are examples. Resistance has been reported to drugs including clotrimazole, 5-fluorocytosine and oligomycin. ${ }^{33}$ There is a theoretical role, therefore, for repeated corneal scrapes in indolent cases to gain sensitivity data and modify therapy if necessary.

Successful medical therapy, with neomycin, propamidine and dibromopropamidine isethionate, was first reported in 1985 by Wright et al. ${ }^{36}$ The diamidines are not, however, freely available in the United States of America and alternative combined therapies have been adopted. Resolution of infection has been achieved with polymyxin-neomycin-gramicidin and miconazole (with and without Brolene) and with neomycin, Brolene and oral ketoconazole (6,37). These represent four cases which did not require adjunctive keratoplasty for control of infection and therapy was instituted around six to 10 weeks after the onset of keratitis.

The regime adopted at Moorfields Eye
Hospital has included hourly topical Brolene and Neomycin initially plus Atropine $1 \%$. The frequency of intensive treatment is generally reduced to two or three hourly during the first week to avoid epithelial toxicity. Three primary referrals with early signs treated in this way, without topical steroids, were clinically resolved within six weeks and no recurrences have occurred after 12 months' follow-up. One case has been lost to follow-up. A further three cases (one bilateral) were tertiary referrals who developed ring infiltrates prior to anti-amoebic treatment. Topical steroids were continued in these cases and the eyes were quiet at three months. Penetrating keratoplasties were performed at this time in two eyes for significant axial opacity and brolene was continued postoperatively. There has been no recurrence after 15 months' follow-up although histopathological examination revealed residual stromal cysts close to the resected margins. The third eye was grafted a year later and remains quiet after six months. Histopathology revealed only IFAb labelled fragments.

Two corneae remained oedematous with persistent epithelial defects, suggesting irreversible endothelial decompensation, despite becoming clinically quiet by three months. Secondary infection occurred in one case at the edge of the defect. Two dense infiltrates extended into adjacent stroma as a crystalline keratopathy (Fig 1). Corneal scraping was performed and both Gram strain and cultures confirmed Streptococcus viridans to be the pathogen. Crystalline keratopathy is an unusual manifestation of bacterial keratitis associated with alpha-haemolytic Streptococcus viridans. It was first described in patients on long term

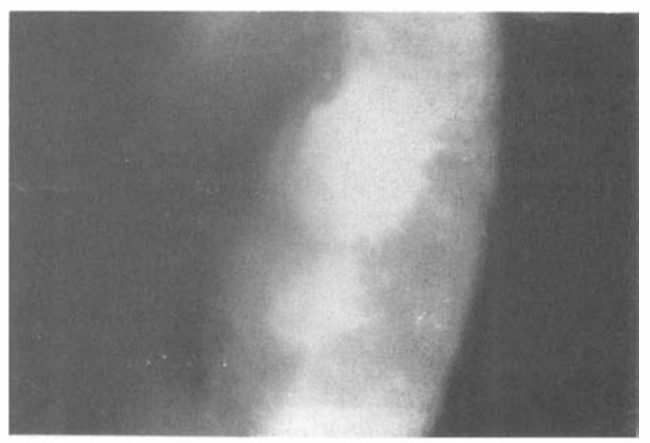

Fig. 1. Crystalline keratopathy due to streptococcus viridans complicating Acanthamoeba keratitis. 


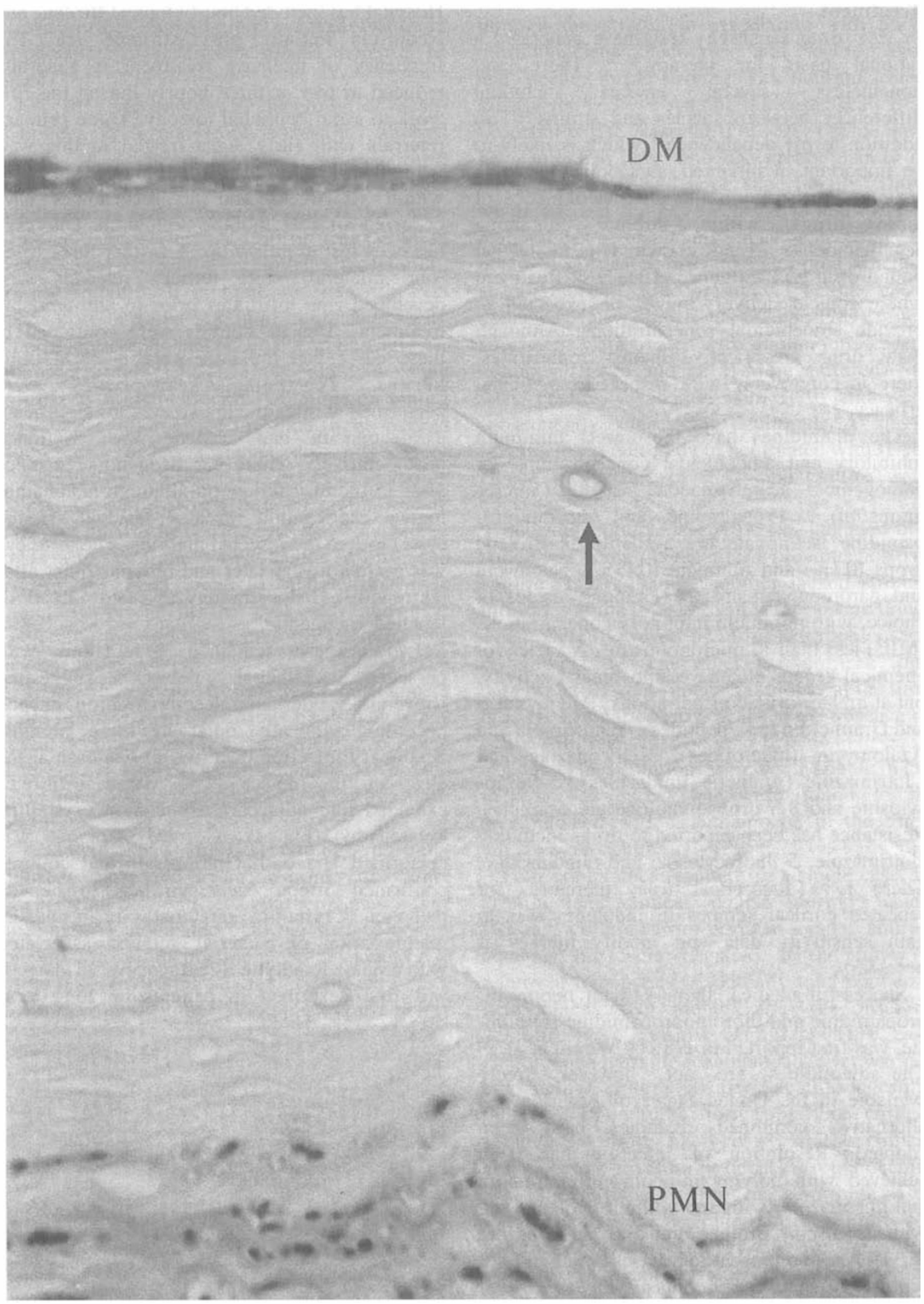

Fig. 2. Histopathological preparation: acanthamoeba cyst is arrowed, deep in the stroma. PMN signifies polymorphonuclear response in the superficial stroma where acanthamoebae are absent. DM signifies Descemet's membrane. 
topical steroid following keratoplasty or herpes simplex keratitis ${ }^{45}$ and more recently in patients with acanthamoeba keratitis. ${ }^{46}$ The keratitis resolved with intensive topical Penicillin G. Although the eye remained quiet, an intumescent cataract required combined cataract extraction with keratoplasty. This graft remains clear at two months. The corneal specimen contained cysts only in the deep stroma which is consistent with acanthamoeba cultures having always been negative (Fig 2). The other case was also grafted and secondary cataract extraction has been necessary. The graft remains clear after nine months' follow-up, although the specimen contained many acanthamoeba cysts. Only these two cases in which anterior segment inflammation had been intense, resulting in cataract formation, also developed secondary glaucoma. Topical timolol maleate $0.25 \%$ and oral acetazolamide controlled intraocular pressure in one case, but the other has required a two-stage Schokett procedure ${ }^{47}$ following a failed trabeculectomy. Glaucoma is a recognised complication and the mechanism may involve direct invasion of the trabecular meshwork since amoebae have been isolated from aqueous ${ }^{48}$ and found in inflammatory membranes adherent to iris of an enucleation specimen. ${ }^{7}$

Secondary infection is a problem. Particularly in cases with unstable or absent epithelium. Since acanthamoeba keratitis can run a fluctuating clinical course, it should not be assumed that recurrent infiltrates are due to acanthamoeba. Streptococcus has been a relatively common secondary pathogen. $6,46,49$

The reported outcome for keratoplasty performed in inflamed and perforated eyes compared with quiet eyes is difficult to interpret since there has not been sufficient follow-up to generate Kaplan-Meier survival curves. ${ }^{50}$ Auran et $\mathrm{al}^{33}$ found useful vision to be slightly more likely following grafting of quiet eyes. Recurrent disease, however, seems to be a feature of cases which were not diagnosed and treated with antiamoebic drugs preopratively and those which were not controlled on treatment and may have developed resistance. ${ }^{1,4,11,12,16,17,26,37}$ Recurrence occurred even where clinically involved tissue had been completely exercised and postoperative treatment was commenced. ${ }^{1,49}$

A bilateral case has run an indolent course following early presentation to Moorfields and remains uncontrolled. He was initially treated with Brolene and Neomycin which resulted in dramatic improvement, but after two months a focal abscess appeared in the region of a previous ring infiltrate. Cultures for acanthamoeba were negative initially. Diffuse stromal infiltration progressed from this focus and acanthamoebae were then identified from a further scrape. Intensive Brolene and Neomycin did not immediately contain this recurrence and sequential treatment with miconazole, Polymyxin B and paromomycin has resulted in a fluctuating course, punctuated by incomplete improvement at times. A penetrating keratoplasty was performed on the left eye, removing all clinically infiltrated stroma, but this failed following early recurrence and subsequently perforated requiring further keratoplasty. The other eye still has stromal infiltration involving predominantly central cornea. In vitro sensitivity tests are being performed to determine the optimal therapeutic regime. This case is the only therapeutic failure for Brolene and Neomycin which therefore remains our treatment of choice.

The role of steroids has been approached experimentally. Osato ${ }^{41}$ found that 15 micrograms $/ \mathrm{ml}$ dexamethasone (attainable in the cornea) inhibits transformation from cyst to trophozoids and vice versa. This may influence susceptibility to anti-amoebic drugs. Blackman et $\mathrm{a}^{43}$ reported the absence of cellular reaction around intact organisms, while there was heavy infiltration by polymorphs and mononuclear cells around necrotic cysts. In the presence of keratocyte necrosis in the vicinity of cysts Garner ${ }^{51}$ has suggested this may be a cytopathic effect of acanthamoeba resulting in secondary leucocyte infiltration and stromal loss. Steroids would then be desirable to reduce this complication. Mathers et $\mathrm{al}^{40}$ interpreted ineffective lymphocyte recruitment in chronic disease to be an expression of macrophage suppression, although immunohistochemistry indicates they are present in large numbers. They were seen to phagocytose cysts, but the absence of activated lymphocytes was thought to explain reduced stimulation of neutrophil function, including elimination of cysts. Clinically, improvement has been achieved with steroids, $2,15-17,36$ deterioration has followed withdrawal 4,9,11,17 and systemic steroids have 
been valuable for nodular scleritis. ${ }^{1,4,6}$ Experience at Moorfields has included control of the disease both with and without steroids.

Following keratoplasty, steroids become important as prophylaxis for graft rejection and many grafts have remained clear when these are combined with anti-amoebic drugs..$^{1,6,40,43,49}$ It is not known how long these should be continued; there are few reported cases of grafted eyes which are no longer on treatment and have no long term follow-up. The cases grafted at Moorfields (1986/1987) have been maintained on topical Brolene twice to four times daily during follow-up periods of up to 16 months.

Acanthamoebic infection is increasingly important in the differential diagnosis of contact lens-related keratitis. The diagnosis is best made using specific cultures and immunofluorescence. Delay influences the diagnostic yield since acanthamoebae may later exist only deep in the stroma, requiring corneal biopsy. Early treatment with topical Brolene and Neomycin is usually effective in controlling infection and reduces the likelihood of recurrence in penetrating grafts for more severe disease. Uncontrolled disease may be managed according to sensitivity data, but recurrent infiltrates should also be investigated for secondary infection. Excision of clinically involved tissue is not a substitute for chemotherapy and graft recurrence is common in cases of progressive disease. More severely inflamed eyes are at risk of glaucoma which may be difficult to control and limit visual recovery. A high index of suspicion is likely to improve the prognosis for this disease.

I would like to thank my colleagues, Mr CM Kirkness, $\mathrm{Mr}$ NSC Rice and Mr P Wright whose patients are included in this report.

My thanks too, to Dr A McCartney for the histopathology and its photography.

\section{References}

${ }^{1}$ Moore MB, McCulley JP, Newton C, Cobo LM, et el. Acanthamoeba keratitis - a growing problem in soft and hard contact lens wearers. Ophthalmology 1987; 94: 1654-61.

2 Jones DB, Robinson NR, Visvesvara GS. Acanthamoeba polyphaga keratitis and Acanthamoeba uveitis associated with fatal meningoencephalitis. Trans Ophthalmol Soc UK 1975;95: 221-32.

${ }^{3}$ Jones BR, McGill JI, Steele ADMcG. Recurrent suppurative kerato-uveitis with loss of eye due to infection by Acanthamoeba castellani. Trans Ophthalmol Soc UK 1975; 95: 210-13.

${ }^{4}$ Mannis MJ, Tamaru R, Roth AM, Burns M, Thirkill C. Acanthamoeba sclerokeratitis. Determining diagnostic criteria. Arch Ophthalmol 1986; 104: 1313-7.

${ }^{5}$ CDC: Acanthamoeba keratitis associated with contact lenses - United States.MMWR 1986; 35: 405-8.

${ }^{6}$ Wilhelmus KR, Osato MS, Font RL, Robinson NM, Jones DB. Rapid diagnosis of Acanthamoeba keratitis using calcofluor white. Arch Ophthalmol 1960; 104: 1309-12.

${ }^{7}$ Ashton N, Stamm W. Amoebic infection of the eye. A pathological report. Trans Ophthalmol Soc UK 1975; 95: 214-20.

${ }^{8}$ Culbertson CG. Soil amoeba infection. Specific indirect immunoenzymatic (peroxides) staining of formalin-fixed paraffin sections. Am J Clin Pathol 1977; 67: 529.

${ }^{9}$ Epstein RJ, Wilson LA, Visvesvara GS, Plourde EG. Rapid diagnosis of Acanthamoeba keratitis from corneal scrapings using indirect fluorescent antibody staining. Arch Ophthalmol 1986; 104: 1318-21.

${ }^{10}$ Robin JB, Chan R, Salazar J. The use of fluorescein-conjugated lectins for the visualisation of Acanthamoebae. Ocular Microbiology and Immunology Group Meeting, Dallas, 1987.

"Cohen EJ, Buchanan HW, Laughrea PA, Adams $\mathrm{CP}$, et al. Diagnosis and management of Acanthamoeba keratitis. Am J Ophthalmol 1985, 100: $389-95$.

${ }^{12}$ Nagington J, Watson PG, Playfair TJ, McGill J, Jones BR, Steele ADMcG. Amoebic infection of the eye. Lancet 1974; 2: 1537-40.

${ }^{13}$ Lund OE, Stefani FH, Dechant W. Amoebic keratitis: a clinicopathologic report. $\mathrm{Br} J$ Ophthalmol 1978; 62: 373-5.

${ }^{14}$ Bos HJ, Volker-Dieben HJM, Kok-van Alphen CC. A case of Acanthamoeba keratitis in the Netherlands. Trans $R$ Soc Trop Med Hyg 1981; 75: 86-91.

${ }^{15}$ Ma P, Willaert E, Juechter KB, Stevens AR. A case of keratitis due to acanthamoeba in New York, and features of 10 cases. $J$ Infect Dis 1981; 143: 662-7.

${ }^{16}$ Hirst LW, Green WR, Merz W, Kaufman C, et àl. Management of Acanthamoeba keratitis. A case report and review of the literature. Ophthalmology 1984; 91: 1105-11.

${ }^{17}$ Theodore FH, Jakobiec FA, Kuechter KB, Ma P, et al. The diagnostic value of the ring infiltrate in acanthamoebic keratitis. Ophthalmology 1985; 92: 1471-97.

${ }^{18}$ Hanssens M, Ded Jonckheere JF, De Meunynck C. Acanthamoeba keratitis. A clinicopathological case report. Int Ophthalmol 1985; 7: 203-13 
${ }^{19}$ Donzis PB, Mondino BJ, Weissmann BA. Microbial contamination of contact lens care systems associated with Acanthamoeba keratitis. Ocular Microbiology and Immunology Group Meeting, Dallas, 1987.

${ }^{20}$ Ludwig IH, Meisler DM, Rutherford I, Bican FE. Susceptibility of acanthamoeba to soft contact lens disinfection systems. Invest Ophthalmol Vis Sci 1986; 27: 626-8.

${ }^{21}$ Kyle DE, Noblet GP. Seasonal distribution of thermotolerant free-living amoebae. I Willard's Pond. J Protozool 1986; 33: 422-34.

${ }^{22}$ Culbertson CG. The pathogenicity of soil amoebas. Ann Rev Microbiol 1971; 25: 231-54.

${ }^{23}$ Brown TJ, Cursons RTM. Pathogenic free-living amoebae from frozen swimming areas in Oslo, Norway. Scand J Infect Dis1977; 9: 237-40.

${ }^{24}$ Griffin JL. Temperature tolerance of pathogenic and non-pathogenic free-living amoebas. Science 1972; 179: 869-70.

${ }^{25}$ Singh BN. Pathogenic and non-pathogenic amoebae. New York: Halsted Press, 1975.

${ }^{26}$ Samples JR, Binder PS, Luibel FJ, Font RI, et al. Acanthamoeba keratitis possibly acquired from a hot tub. Arch Ophthalmol 1984; 102: 707-10.

${ }^{27}$ Cursons RTM, Brown TJ, Keys EA. Immunity to pathogenic free-living amoebae. Lancet 1977; 1: 875-6.

${ }^{28}$ Wang SS, Feldman HA. Isolation of Hartmanella species from human throats. N Eng J Med 1967; 277: 1174-9.

${ }^{29}$ Lawande RV, Abraham SN, John I, Egler LJ. Recovery of soil amoebas from the nasal passages of children during the dusty haramattan period in Zaria. Am J Clin Path 1979; 71: 201-3.

${ }^{30}$ Bandenoch PR, Grimmond TR, Cadogan J, Deayton SE, Essery MSL, Hill BD. Nasal carriage of free-living amoebae. Microbiol Ecology Health Dis. (In Press.)

${ }^{31}$ Martinez AJ. Acanthamoebiasis and immunosuppression. J Neuropathal Exp Neurol 1982; 41: 548-57.

32 Gonzalez MM, Gould E, Dickman G, Martinez AJ, et al. Acquired immunodeficiency syndrome associated with Acanthamoeba infection and other opportunisitic organisms. Arch Path and Lab Med 1986; 110: 749-51.

${ }^{33}$ Auran JD, Starr MB, Jakobiec FA. Acanthamoeba keratitis. A review of the literature. Cornea 1987; 6: 2-26.

${ }^{34}$ Moore MB, McCulley JP, Luckenbach MD, Gelander $\mathrm{H}$, et al. Acanthamoeba keratitis associated with soft contact lenses. $\mathrm{Am} J$ Ophthalmol 1985, 100: 396-403.

35 Yeoh R, Warhurst DC, Falcon MG. Acanthamoeba keratitis. $\mathrm{Br} J$ Ophthalmol 1987; 71: 500-3.

${ }^{36}$ Wright P, Warhurst DC, Jones BR. Acanthamoeba keratitis - successfully treated medically. $\mathrm{Br} J$ Ophthalmol 1985; 69: 778-82.
${ }^{37}$ Moore MB, McCulley JP, Kaufman HE. Presentation at Ocular Microbiology and Immunology Group Meeting, San Francisco, 1985.

${ }^{38}$ Culberton CG, Ensminger PW, Overton WM. Hartmanella. Experimental chronic, granulomatous brain infections produced by new isolates of low virulence. Am J Clin Pathol 1966; 46: 305-14.

${ }^{39}$ Osato MS, Robinson NM, Wilhelmus K, Jones DB. Morphogenesis of Acanthamoeba castellannii. Titration of steroid effect. Invest Ophthalmol Vis Sci 1986; 27 (suppl): 37.

${ }^{40}$ Mathers W, Stevens G, Rodrigues M, Chan CC, et al. Immunopathology and electron microscopy of Acanthamoeba keratitis. Am J Ophthalmol 1987, 103: 626-35.

${ }^{4 l}$ Osato MS, Robinson NM, Wilhelmus KR, Jones DB. Interpretations of tests for acanthamoeba keratitis: pseudo-acanthamoebic trails. Ocular Microbiology and Immunology Group Meeting, Dallas, 1987.

42 Visvesvara GS, Jones DB, Robinson NM. Isolation identification and biological characterisation of acanthamoeba polyphaga from a human eye. $\mathrm{Am}$ J Trop Med Hyg 1975; 24: 784-90.

${ }^{43}$ Blackman HJ, Rao NA, Lemp MA, Visvesvara GS. Acanthamoeba keratitis successfully treated with penetrating keratoplasty: suggested immunogenic mechanisms in action. Cornea 1984; 3: 125-30.

${ }^{44}$ Nagington J, Richards JE. Chemotherapeutic compounds and acanthamoeba from eye infections. J Clin Pathol 1976, 29; 648-51.

${ }^{45}$ Meisler DM, Langston RHS, Naab TJ. Infectious crystalline keratopathy. $A m \quad J$ Ophthalmol 1984;97: 337-43.

${ }^{46}$ Davis RM, Schroeder RP, Rowsey JJ, Jensen HG, Tripathi RC. Acanthamoeba keratitis and infectious crystalline keratopathy. Arch Ophthalmol 1987; 105: 1524-7.

${ }^{47}$ Kirkness CM. Penetrating keratoplasty, glaucoma and silicone drainage tubing. Developments in Ophthalmology. Karger, Basel, 1987; 14: 165-9.

${ }^{48}$ McClellan K, Coster DJ. Acanthamoeba keratitis diagnosed by paracentesis and biopsy and treated with propamidine. $\mathrm{Br} J$ Ophthalmol 1987; 71: 734-6.

${ }^{49}$ Cohen EJ, Perlato CJ, Arentsen JJ, Genvert GI, et al. Medical and surgical treatments of acanthamoeba keratitis. Am J Ophthalmol 1987; 103: 615-25.

${ }^{50}$ Kaplan EL, Meier P. Non-parametric estimation from incomplete observations. Am J Stat Assoc 1958; 53: 457-81.

${ }^{51}$ Garner A. Pathogenesis of Acanthamoeba infections. Proc World Congress on the Cornea III, Cornea. (In Press.) 